\title{
Commissioning of Electromechanical Conversion Models for High Dynamic PMSM Drives
}

\author{
Silverio Bolognani, Member, IEEE, Luca Peretti, Student Member, IEEE, Mauro Zigliotto, Member, IEEE and
} Ezio Bertotto

\begin{abstract}
Several emerging applications require fast and precise torque control. Torque measurements are still expensive, bulky and delicate. On the other hand, estimation techniques are all valid in principle, but their accuracy is largely affected by model identification and commissioning. This paper presents an accurate model for an effective torque estimation based on voltage and current measurements. The model includes all motor losses to get an enhanced overall accuracy, along with a fast response. The key-feature is the commissioning procedure, based on a set of offline measurements. Procedure details and experimental results on a laboratory prototype are included.
\end{abstract}

Index Terms-AC motor drives, Permanent magnet motors, Parameter estimation, Torque control.

\section{INTRODUCTION}

$\mathbf{T}$ HE estimation of either the electromechanical or shaft torque is arousing living interest in many advanced drives applications. While the study of torque components and their dependence on motor geometry is a key issue for an improved motor design [1], the availability of instantaneous torque estimation is useful in manifold applications, as for example in high-power applications where the shaft torque transducer is both expensive and difficult to place and maintain [2], in sensorless direct torque control [3], and in health care applications [4], [5]. In [6] the precise torque value is necessary for proper ripple compensation in a sensorless brushless DC motor. The solution is based on the conventional expression of the electromagnetic torque, and it relies on the knowledge of both speed and back-electromotive force, which are obtained by a simplified Kalman filter and a sliding mode observer respectively. Similarly, in [7] the instantaneous torque value is obtained by a MRAS technique to reduce torque pulsation due to flux harmonics while to the same aim a gainshaped sliding-mode observer is used in [8] to implement iterative learning torque control. The results are effective, with good torque estimation accuracy. Anyway, the estimation does include neither iron losses, which are not negligible

Manuscript received ...; revised ... .

Copyright (C) 2009 IEEE. Personal use of this material is permitted. However, permission to use this material for any other purposes must be obtained from the IEEE by sending a request to pubs-permissions@ieee.org.

S. Bolognani is with the University of Padova, Department of Electrical Engineering, Via Gradenigo 6/A, 35131 Padova, Italy (e-mail: silverio.bolognani@die.unipd.it).

L. Peretti and M. Zigliotto are with the University of Padova, Department of Technique and Management of Industrial Systems, Stradella San Nicola 3, 36100 Vicenza, Italy (e-mails: luca.peretti@unipd.it, mauro.zigliotto@unipd.it).

E. Bertotto is with REEL srl, via Riviera Berica 42, 36024 Ponte di Nanto (Vicenza), Italy (e-mail: bertotto@ reel.it). especially for high-power drives, or mechanical losses, which are important to derive the net shaft torque.

Another example is reported in [9], where the highly nonlinear electromagnetic flux-current-angle and torque-currentangle relationships of a switched reluctance motor are obtained by a Cerebellar Model Adaptive Control (CMAC), and an adaptive B-Spline network gives real-time torque estimation. For high speed operation however, CMAC requires a FPGA hardware implementation, and the B-spline needs a TMS320C6711 DSP as a co-processor for a TMS320F243 DSP controller. Therefore, the computational requirements are heavy, and the estimate still suffers of the accuracy of the motor electromagnetic model. A fair example of torque estimate for induction motors is reported in [10]. The algorithm is based on stator voltage and machine current measurements. The stator flux synthesis, which leads to the torque estimation, presents the tricky problem of the offset in the time integral. The digital implementation is performed by a Programmable Cascaded Low-Pass Filters [11], implemented by using Recurrent Neural Network (RNN-PCLPF) trained by an algorithm based on Kalman filter. The precision falls within the $2 \%$, even if iron and additional losses are not included, which are generally not negligible, especially in large motor drives.

Latest research strategies, successfully applied to the torque estimation of internal combustion engines, are based on the stochastic approach and described in [12] and [13]. This approach, which is based on the correlations between incylinder pressure and crankshaft speed variations, gives accurate estimations of the instantaneous in-cylinder pressure, with light computational requirements. It is believed that the method may have very high potential also for application in electrical motors.

Anyway, and despite the well-motivated interest of industry about the topic, evidently the ultimate solution has not been proposed yet. The precise electromechanical conversion model plays a crucial role in the torque estimate, and it involves manifold aspects, like specific motor and drive design, available measurements, signal accuracy, numerical precision and computational requirements. For all of that, it is acceptable that a precise model is customised on the application. Nevertheless, to our experience, an accuracy below 3-4\%, referred to the nominal torque on wide speed and load ranges, is still a charming challenge in most cases.

This paper proposes an innovative commissioning procedure and a simple scheme for torque estimation in a permanent magnet synchronous motor (PMSM) drive. The estimation is model-based and it relies on both phase currents and voltages measurement, which are still very delicate items. A scheme 
for precise voltage acquisition has been specifically developed and tested on the field. As detailed in the paper, the estimation model is not affected by magnetic saturation nor uses any magnetic model of the motor, which is not handy and does not assure enough precision especially for high power drives.

The motor parameters used in the estimation model are obtained by means of the commissioning procedure, which is performed off-line by a series of standard measurements followed by a specific data post-processing. The paper includes experimental results, which confirm the effectiveness of the procedure. Eventually, the accuracy of the torque estimation has been verified by comparison with a wide-bandwidth highprecision torquemeter, coupled to the motor shaft.

\section{THE MOTOR MODEL}

Conventional vector control strategies are performed under the assumption that there are no iron losses in the motor. However, since the iron losses influence both the flux linkage and electromagnetic torque, they have been necessarily included in the motor model. Several Authors have made an attempt to consider the iron losses in vector-controlled $\mathrm{AC}$ motor drives [14], [15], [16]. In those studies, iron losses are modelled as the thermal power dissipated on an "iron loss" resistance placed in series, or in parallel, to the conventional PMSM equivalent circuits. From a modelling point of view, it has been found that there is substantial equivalence between series and parallel types [17]. Hereafter, the latter will be chosen for its better adherence to the physical meaning [17]. The PMSM voltage balance equations in a stationary $\alpha-\beta$ reference frame are the following:

$$
\begin{aligned}
& u_{\alpha}=R_{s} i_{\alpha}+\frac{d \lambda_{\alpha}}{d t} \\
& u_{\beta}=R_{s} i_{\beta}+\frac{d \lambda_{\beta}}{d t}
\end{aligned}
$$

where $R_{s}$ is the stator resistance and $\lambda_{\alpha}, \lambda_{\beta}$ are the flux linkages. The related PMSM equivalent circuits are reported in Figure 1.

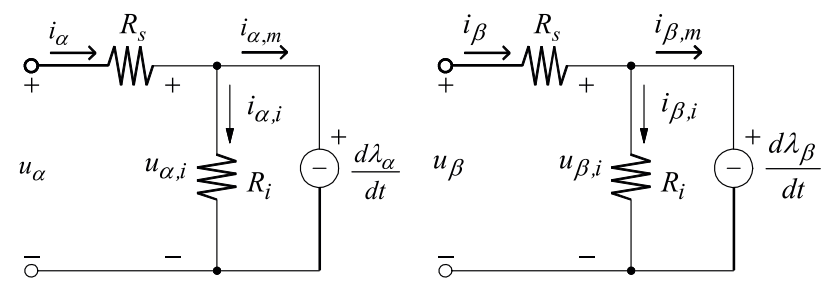

Figure 1. PMSM $\alpha-\beta$ equivalent circuits, including iron losses.

The iron losses equivalent resistance $R_{i}$ is inserted in parallel with each magnetizing branch. Thus, the axis currents $\left(i_{\alpha}, i_{\beta}\right)$ are divided into the iron loss currents $\left(i_{\alpha, i}, i_{\beta, i}\right)$ and magnetizing currents $\left(i_{\alpha, m}, i_{\beta, m}\right)$ :

$$
\begin{aligned}
i_{\alpha, i} & =G_{i} \frac{d \lambda_{\alpha}}{d t} \\
i_{\beta, i} & =G_{i} \frac{d \lambda_{\beta}}{d t}
\end{aligned}
$$

where $G_{i}=1 / R_{i}$ is the iron loss conductance which is, in general, a non-linear function of the electrical angular speed $\omega_{m e}[15]$

\section{TORQUE ESTIMATION ALGORITHM}

Since both the copper losses and the iron losses are explicitly considered, the remaining is a conservative system in which the electromagnetic torque can be obtained by the cross-product of the direct and quadrature flux linkages by the magnetizing currents:

$$
\tau_{e}=\frac{3}{2} p\left(\lambda_{\alpha} i_{\beta, m}-\lambda_{\beta} i_{\alpha, m}\right)
$$

where $p$ represents the pole pairs of the PMSM. Expressions (1)-(3) describe the electromechanical torque estimator algorithm, which is outlined in Figure 2.

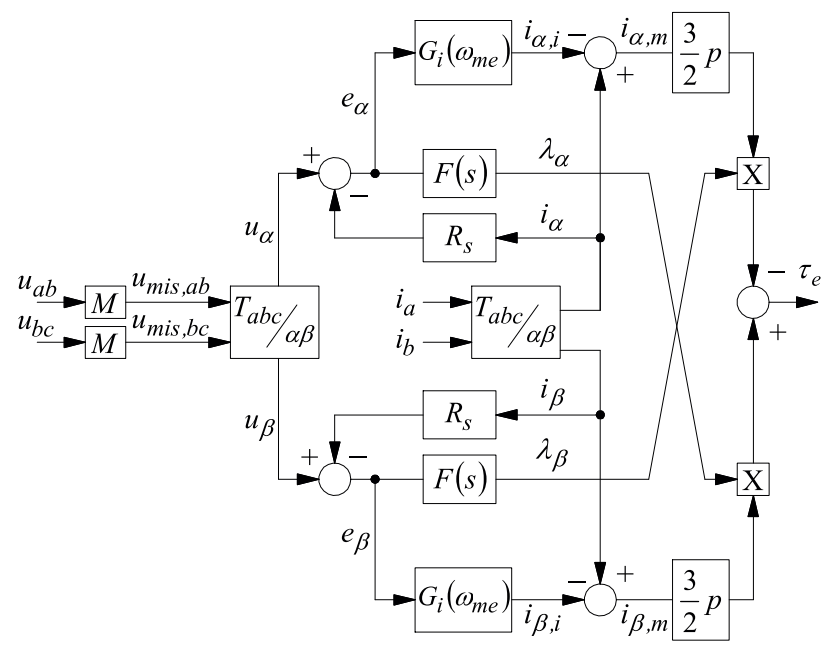

Figure 2. Block schematic of the torque estimator.

$M$ represents a custom measurement block for phase-tophase voltages, while $F(s)$ represents a special integration block. Details on both blocks will be given in the next subsections. $T_{a b c / \alpha \beta}$ is the conventional three-to-two-phases stationary coordinate transformation used for both measured voltages and currents. The proposed scheme should be used from motor startup, in order to start from zero initial conditions for both $\lambda_{\alpha}$ and $\lambda_{\beta}$. It accounts for saturation effects, since the integration can be computed from voltage and current measurements without the knowledge of any flux linkage saturation model. Afterwards, the shaft torque will be derived by subtracting an estimation of the mechanical and additional torque losses from the electromagnetic torque shown in (3) and Figure 2. Next sections will describe the offline commissioning procedure which will produce the parameters $R_{s}, G_{i}\left(\omega_{m e}\right)$ that will be used in the scheme of Figure 2 to get real time torque estimation for drive control purposes.

\section{A. Voltage measurements}

Despite its simplicity, the accuracy of the scheme directly depends upon the quality of voltage measurements. To this purpose, the block $M$ was specifically developed in this work. It consists of two analog integrators connected in parallel. The principle of operation is reported in Figure 3 for the $u_{a b}$ voltage, and the same holds for $u_{b c}$.

A synchronism signal (sync) is derived from the PWM generator, at a rate equal to the switching frequency $1 / T_{c}$. A 


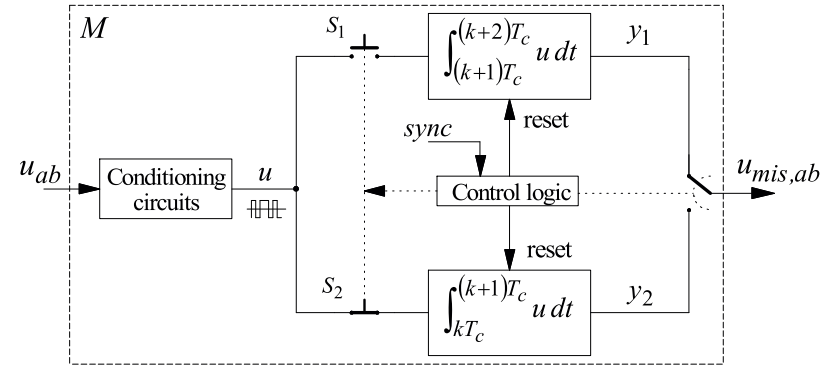

Figure 3. Block schematic of the voltage measurement circuit.

sequential control logic manages the input to the integrators through solid-state switches $S_{1}$ and $S_{2}$. Each integrator gives the mean value of the voltage within a switching period. While one integrator is working, the other is reset, and its output is tied up to the output $u_{m i s, a b}$. The use of integrators in parallel yields a zero-delay swap, which is fundamental for the accuracy of the measurement. High bandwidth, high slew-rate components assure the accuracy of integration. The passive elements have been manually matched to improve the overall precision, which is around $1 \%$. Some circuital solutions, as self-compensating topologies, also guarantee a low sensitivity to temperature variations.

It is worth to note that the proposed circuit preserves the low-frequency content of the voltage signal, while deleting the contribution at the switching frequency and its harmonics. The same holds for the phase currents, which are sampled synchronously with the switching period, to skip aliasing effects. Thus, actual voltages and currents do not fit for direct derivation of PWM losses, which are sometimes non negligible, as discussed in [18] and reported later in the paper.

\section{B. Flux linkage integration}

In principle, the flux linkages are calculated by pure integration of their own derivatives, obtained from the phase voltages (1). The unavoidable presence of input offset makes this solution unfeasible. An acceptable trade-off between algorithm complexity and effectiveness was found to be a cascade of a low-pass filter and a high-pass filter, which gives an overall transfer function

$$
F(s)=\frac{s \tau^{2}}{(1+s \tau)^{2}}
$$

The proposed function approximates the ideal integrator for frequencies above $30 \mathrm{~Hz}$, as shown in Figure 4.

As well, the torque estimator will work properly above that limit, which was considered acceptable. To increase integration accuracy, the phase and magnitude distortions introduced by $F(s)$, as function of frequency, have been stored in lookup table and properly compensated on-line. Anyway, when low-speed torque estimation is required, a modified integrator with adaptive compensation [19] or cascaded low-pass filterbased techniques [11] should be used instead, at the cost of an increased system complexity.

\section{THE COMMISSIONING PROCEDURE}

The commissioning procedure plays a crucial role in the successful estimation of stator and iron losses resistances and
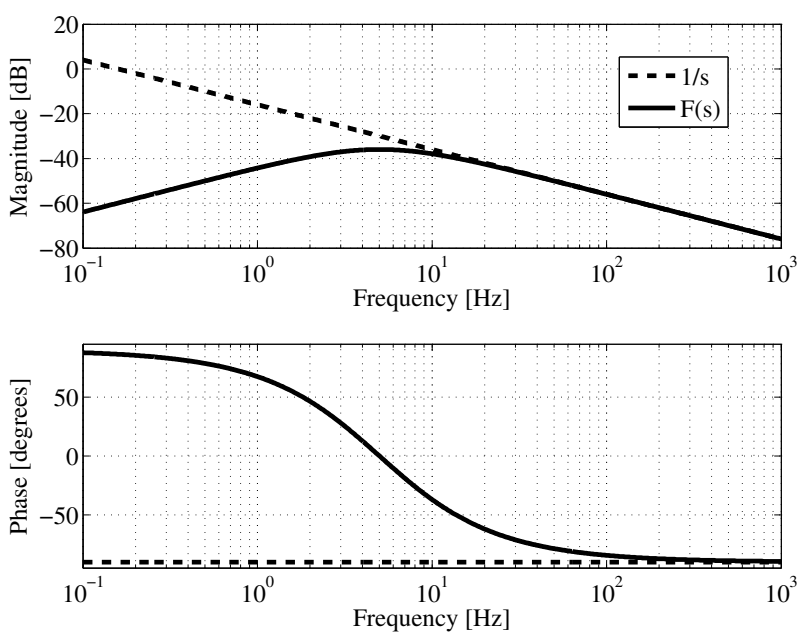

Figure 4. Bode diagram of pure integrator and proposed implementation.

the related tuning of the torque estimator of Figure 2. Figure 5 reports a sketch of the experimental setup.

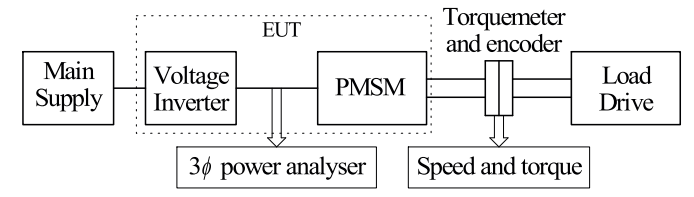

Figure 5. Experimental setup for preliminary power measurements.

The procedure consists of two main steps, both performed off-line. The first one is a series of power measurements, under specified working conditions. The input power $P_{i n}$ was measured by a LEM NORMA 4000 power analyser, which is especially suitable for on-site use. It also gives current and voltage with high accuracy and calculates active, reactive and apparent power and other derived values. Line-to-line voltages were measured directly, while phase currents were acquired by external probes. The instrument's accuracy is of $0.1 \%$ and its bandwidth of DC to $3 \mathrm{MHz}$. The load is represented by a second drive (Figure 5) that can be either speed or torque regulated.

The second step of the proposed commissioning procedure is a post-elaboration of the collected data, according to a sequence that is committed to extract the desired parameters and their dependence upon operating conditions.

\section{A. Power measurements}

The first batch of measurements was obtained at constant speed and current amplitudes, for different torque levels. The mechanical speed $\omega_{m}$ was imposed by the load drive (Figure 5 ), in constant speed mode. Actually, the drive under test (EUT) is therefore operated in braking mode, and negative torque values $(\tau<0)$ are expected. The experiments were then repeated at different speed. Table I reports an example of data collected for a mechanical speed of 0.6 p.u., and nominal line current. The base quantities for the calculation of the p.u. values of the used motor are reported in the Appendix. 
Table I

MEASUREMENTS AT CONSTANT SPEED AND CURRENT (P.U. VALUES).

\begin{tabular}{c|c|c|c|c}
\hline Torque $\tau$ & -0.508 & -0.772 & -0.814 & -0.817 \\
\hline Input power $P_{\text {in }}$ & -0.292 & -0.447 & -0.472 & -0.473 \\
\hline Line-to-line voltage $V$ & 0.294 & 0.450 & 0.505 & 0.549 \\
\hline Total losses $P_{L}$ & 0.013 & 0.016 & 0.016 & 0.017 \\
\hline
\end{tabular}

Table II

MEASUREMENTS AT CONSTANT SPEED AND CURRENT (P.U. VALUES).

\begin{tabular}{c|c|c|c|c}
\hline Torque $\tau$ & -0.468 & -0.578 & -0.666 & -0.806 \\
\hline Input power $P_{i n}$ & -0.273 & -0.337 & -0.388 & -0.468 \\
\hline Phase current $I 0.468$ & 0.582 & 0.677 & 0.833 & \\
\hline Total losses $P_{L}$ & 0.008 & 0.010 & 0.012 & 0.016 \\
\hline
\end{tabular}

For each measurement, the total power loss was calculated as the difference between the input power and the mechanical power at the motor shaft:

$$
P_{L}=\left|P_{i n}-\omega_{m} \tau\right|
$$

A second batch was performed at constant speed and flux linkage. The latter was evaluated from the integration of the back electromotive forces $e_{a}$ and $e_{b}$, which is a part of the torque estimator that can be used before its complete commissioning, as it appears from Figure 2. Again, the measurements were repeated for different speeds, up to the nominal one. Table II reports an example of p.u. data collected at nominal flux linkage.

The collected data were then post-processed to complete the model commissioning, as detailed in the next sections.

\section{B. Evaluation of the stator resistance}

For large power drives the measure of the stator resistance with conventional instrumentation is often impractical. Here a viable procedure is proposed instead. At constant flux linkage the iron losses are supposed to be constant. The slope of the linear interpolation of the power losses as function of the square current then returns a resistance value for each set of measurements. The slopes, i.e. the resistances, obtained for different speeds are then plotted as function of speed itself. It has been found that a linear dependence can be obtained by considering the square root of the speed, as reported in Figure 6.

The equation of the interpolating straight line is

$$
R\left(\omega_{m e}\right)=k_{1} \sqrt{\omega_{m e}}+R(0)=k_{1} \sqrt{\omega_{m e}}+R_{s}
$$

where $k_{1}=0.016$ [p.u.] and $R_{s}=0.00476$ [p.u.]. The intercept $R(0)$ is taken as (constant) stator resistance. The remaining speed-dependent losses are mainly due to the skin and proximity effects which cause an asymmetrical distribution of the current in the stator winding paths, and they will be considered later on, as part of the additional losses. It is worth to note that the linear relationship of (6) have been sought by means of a manual search. Different motors could call for a different choice of the $x$ axis variable to get, when possible, a linear relationship that eases the extrapolation.

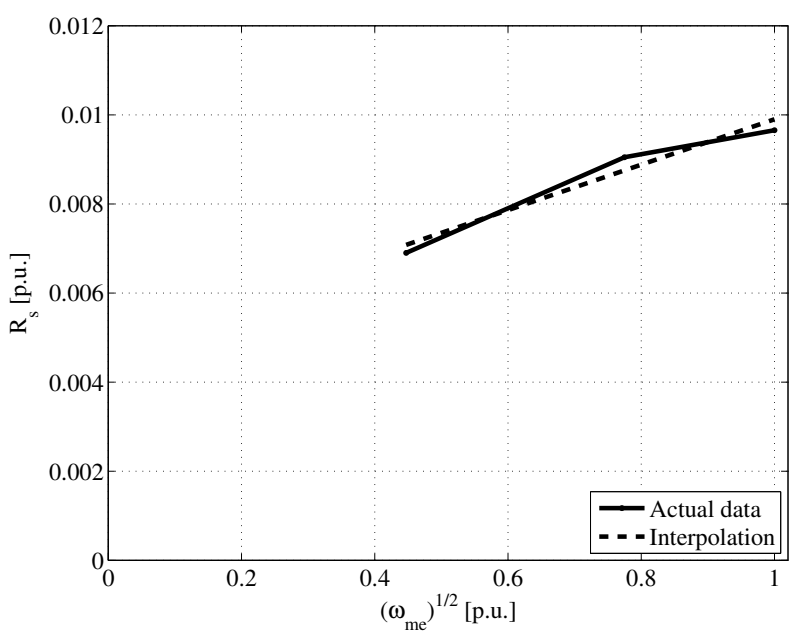

Figure 6. Stator resistance vs. square root of the speed.

\section{Iron losses model commissioning}

According to the schemes of Figure 1, the iron losses are modelled as the power dissipated on the conductance $G_{i}\left(\omega_{m e}\right)$. The commissioning procedure exploits the data obtained at constant speed and current (Table I), so that copper, mechanical and additional losses can be considered altogether as a constant $P_{L, 0}$. The total losses can be expressed as the sum of $P_{L, 0}$ and a term that is a linear function of the square of the rms voltages $u_{\alpha, i}, u_{\beta, i}$ (Figure 1):

$$
\begin{aligned}
P_{L, i} & =G_{i}\left(\omega_{m e}\right)\left(u_{\alpha, i r m s}^{2}+u_{\beta, i r m s}^{2}\right)+P_{L, 0} \\
& =G_{i}\left(\omega_{m e}\right)\left|u_{i r m s}\right|^{2}+P_{L, 0}
\end{aligned}
$$

where $\left|u_{i r m s}\right|$ is the rms value of the phase voltages. It can be derived from the measurements of Table I, completed with the information about the actual phase currents readily available from the current control system of the drive. The iron losses as function of the square voltage $\left|u_{i r m s}\right|^{2}$ at three different speeds have been calculated, as shown in Figure 7, Figure 8 and Figure 9.

The angular coefficients of each of the straight lines of Figure 7, Figure 8 and Figure 9 represent a value of the conductance $G_{i}\left(\omega_{m e}\right)$. The proper choice of the $x$ axis coordinate (in this case as shown in Figure 10, the reciprocal of the speed) lets the conductance $G_{i}\left(\omega_{m e}\right)$ to be a straight line, so that:

$$
G_{i}\left(\omega_{m e}\right)=\frac{m_{i}}{\omega_{m e}}
$$

with $m_{i}=0.011$ [p.u.].

\section{Additional losses model commissioning}

Additional losses causes are manifold, and conceptually they cannot be attributed to a single source. They include, but are not limited to, both PWM losses and skin effects.

The losses related to the skin effect vary with the square root of frequency. For practical PWM current ripples of 20-30\% of rated current, copper losses augment due to PWM, even accounting for skin-effect, is estimated around $10-15 \%$, that alone cannot explain significant total PWM loss. It means that 


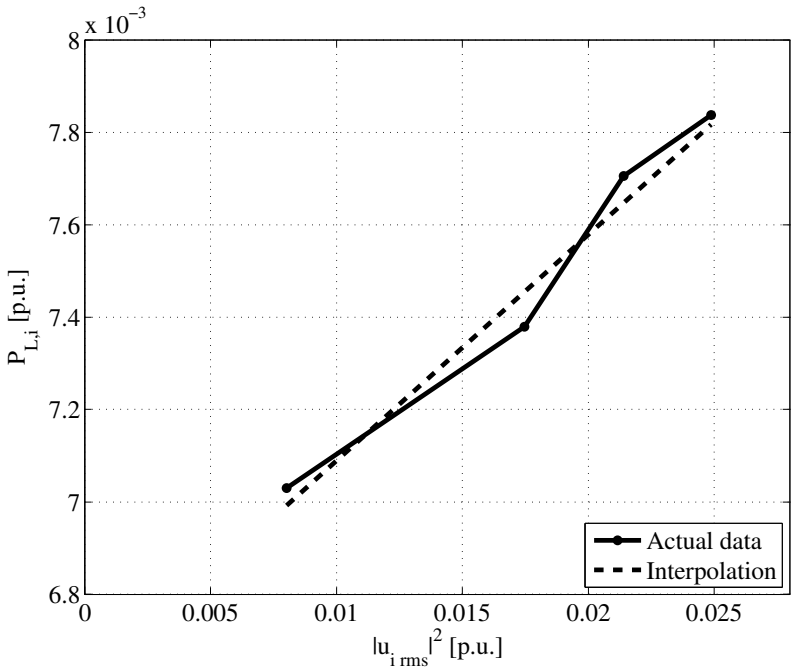

Figure 7. Total losses $P_{L, i}$ as function of square voltage ( $\omega_{m}=0.2$ p.u.).

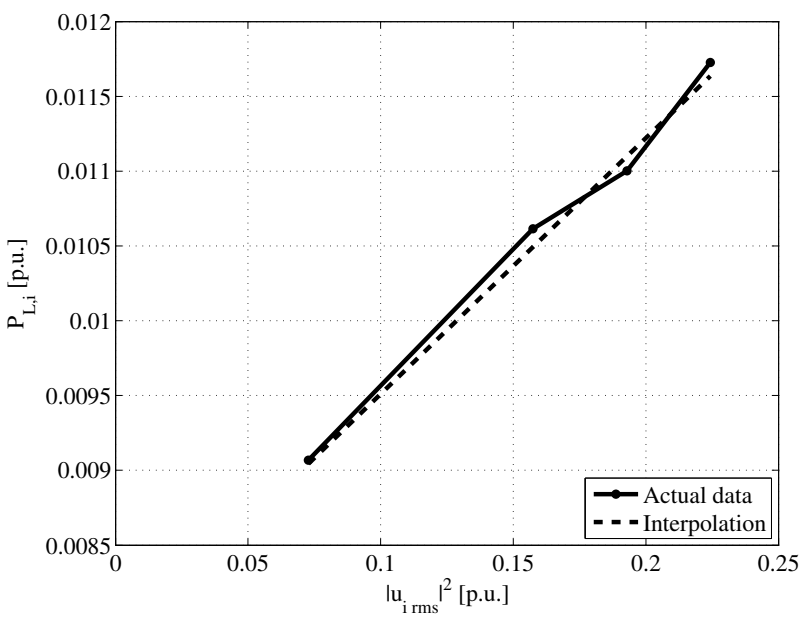

Figure 8. Total losses $P_{L, i}$ as function of square voltage $\left(\omega_{m}=0.6\right.$ p.u. $)$

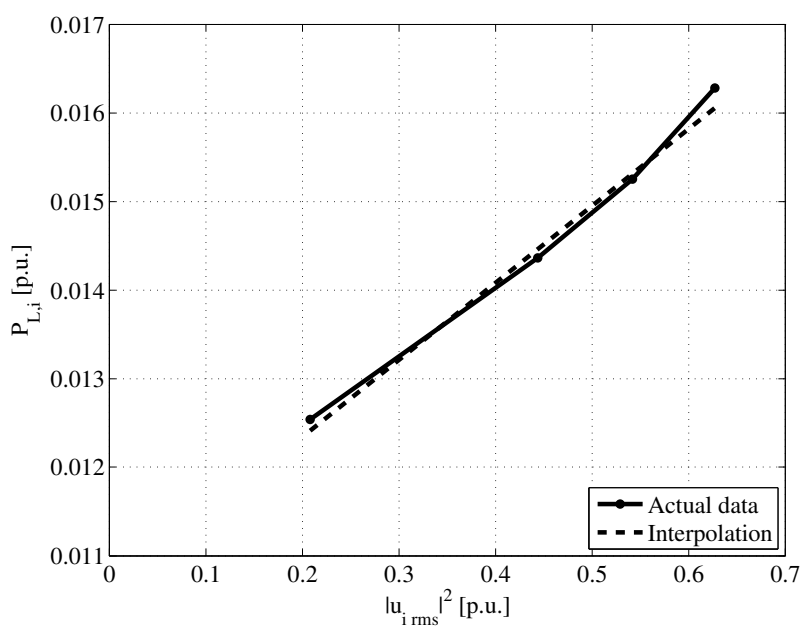

Figure 9. Total losses $P_{L, i}$ as function of square voltage $\left(\omega_{m}=1\right.$ p.u. $)$.

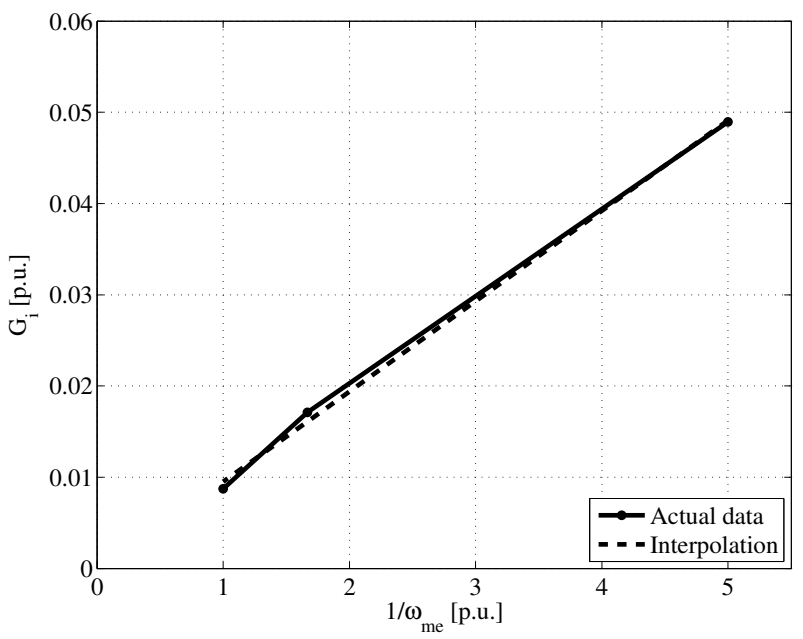

Figure 10. Iron losses conductance $G_{i}$ as function of speed reciprocal.

PWM core loss is a dominating mechanism. For PM machines, PWM eddy current losses are supposed to take place mostly in PWM excited armature yoke, because of relatively large equivalent air gap. Some PWM losses are also expected in permanent magnet alloy materials that, as opposed to ferrites, have relatively high conductivity. Accurate and detailed studies have been reported by different Authors, for example [18].

This paper adopts a different approach. Additional losses are not tentatively evaluated from equations based on the probable physical origin of each loss term as in [16]. Instead, joined additional and mechanical losses $P_{a}+P_{\text {mech }}$ are derived as the difference between total measured losses $P_{L}$ and both iron and copper losses ( $P_{L, i}$ and $P_{L, c u}$, respectively).

At constant speed and flux linkage (Table II), constant iron losses can be assumed. $P_{a}+P_{\text {mech }}$ are plotted as function of the square amplitude of the phase currents. Fig. 9 reports the plot for $\omega_{m}=0.6$ p.u..

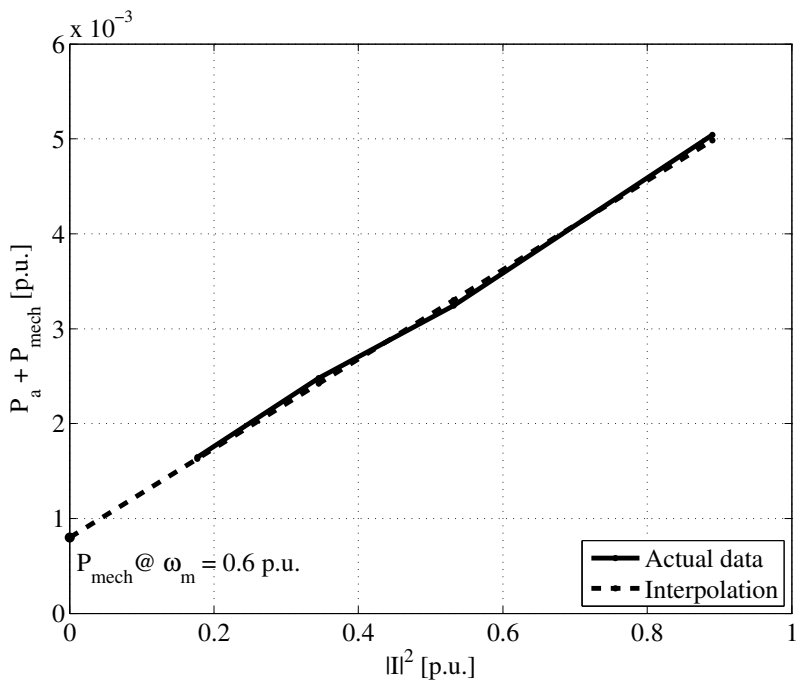

Figure 11. Mechanical and additional losses as function of square current, at constant speed and flux linkage.

Similar plots have been drawn at different speeds, resulting 
in as much straight lines. The intercepts represent the losses at no current, which can be related to mechanical losses. It is possible to elaborate a polynomial approximation of the mechanical losses as function of the speed. In the present work, it has been found that the mathematical link between mechanical losses and speed is well defined by the expression

$$
P_{m e c h}\left(\omega_{m e}\right)=k_{1} \omega_{m e}^{4}+k_{2} \omega_{m e}^{2}
$$

where $k_{1}$ and $k_{2}$ are constant coefficients. Once $P_{\text {mech }}$ has been evaluated, it can be subtracted from $P_{a}+P_{\text {mech }}$ to obtain an estimation of the remaining additional losses. The latter are in general a non-linear function of both the current level and the speed. Different approaches can be adopted to model the additional losses. A possible way is to derive the additional losses in an adequate number of working points, and store the results in look-up tables (LUTs). Of course, the accuracy is affected by LUTs resolution and their capability of covering the whole drive working conditions. An alternative, successfully adopted in this work, is to investigate a possible explicit relationship between additional losses and both speed and current. It has been found, as shown in Figure 12, that there exists a linear relation between $\mathrm{Pa}$ divided by the square current amplitude and the reciprocal of the squared speed.

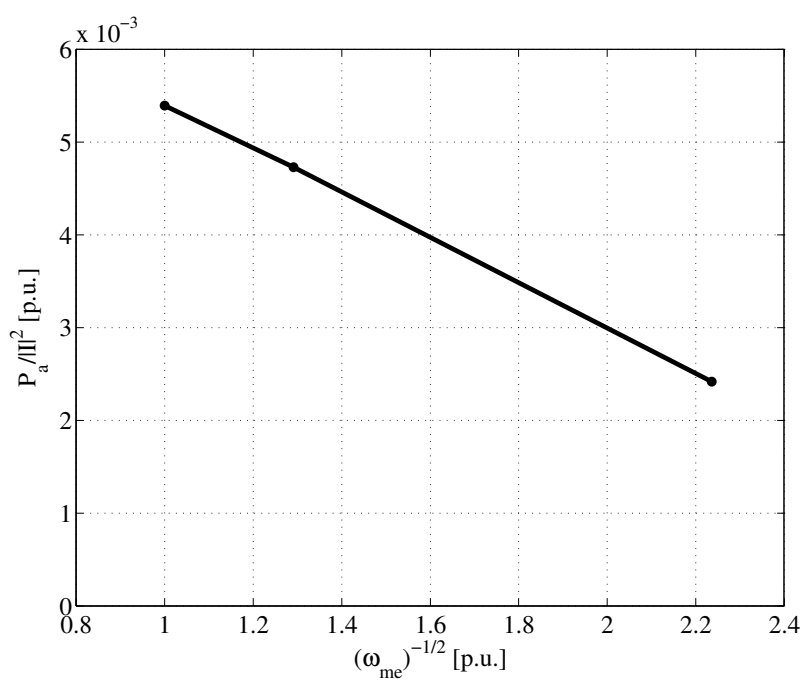

Figure 12. Additional losses as function of speed and current.

The non-linear function that has been used to model additional losses is therefore

$$
P_{a}=\left(\frac{k_{3}}{\sqrt{\omega_{m e}}}+k_{4}\right)|I|^{2}
$$

where $k_{3}$ and $k_{4}$ are constants that can be directly derived from Figure 12. It is worth to note that this polynomial approximation is the mathematical representation of complex phenomena which strongly depend upon the considered motor and mechanical coupling. Other experimental setups could require different expressions. This fact should not represent a problem. Actually, the proposed algorithm is meant to be a substitute of a torque transducer in high-hand applications, which are usually prone to ad-hoc commissioning.
The additional and mechanical losses are turned into a torque loss by dividing them by the mechanical speed. The torque loss is then properly subtracted to the estimated electromechanical torque $\tau_{e}$ of (3) to get the estimation of the net shaft torque $\tau_{s}$ :

$\tau_{s}=\tau_{e}-p\left(k_{1} \omega_{m e}^{3}+k_{2} \omega_{m e}+k_{3}|I|^{2} \omega_{m e}^{-3 / 2}+k_{4}|I|^{2} \omega_{m e}^{-1}\right)$

\section{EXPERIMENTAL RESULTS}

The torque estimator of Figure 2 has been implemented on a laboratory prototype for validation. The motor, whose parameters are shown in Appendix, was fed by a SVM inverter with symmetric PWM at $10 \mathrm{kHz}$. Current control was performed by conventional PI regulators with axis decoupling and back-electromotive force feedforward. The PMSM motor was a prototype with isotropic structure and surfacemounted magnets. The commissioning has been obtained by the procedure outlined in this paper, tuning the estimator with the requested motor parameters. The shaft torque has been measured with a high-precision torquemeter, and compared with the torque estimated by (11). Figure 13 reports the relative estimation error, as function of the motor speed, for different load conditions.

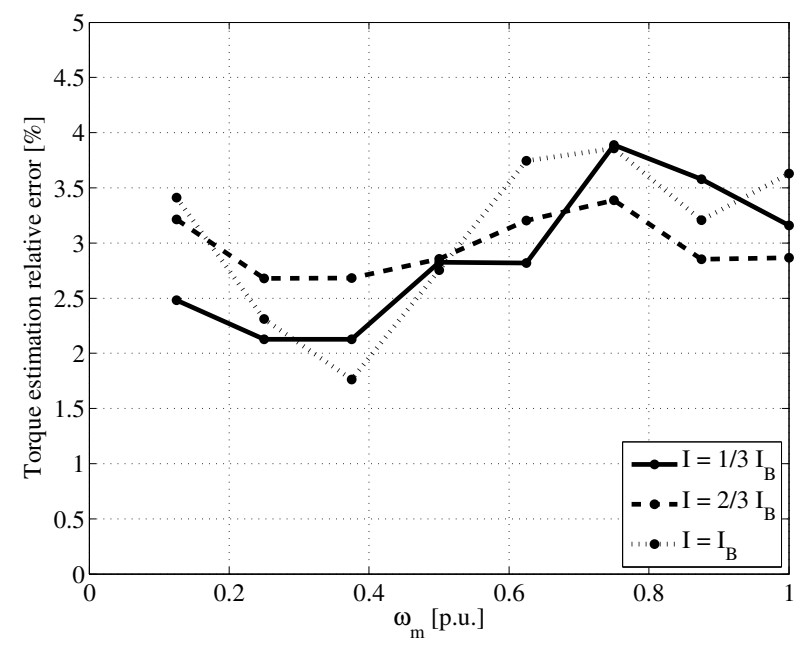

Figure 13. Torque estimation relative error vs. speed and current.

It is worth to note that the error remains within $4 \%$ in the whole speed and torque range of the drive. The error is relative to the actual measured torque, thus the reference to the nominal torque value would still improve the figures. The error is always of the same sign, which suggests the possible presence of a systematic error, which will be further investigated. A possible source of error could be a parameter mismatch due to non-perfect commissioning procedure.

While accounting for all possible losses, and thus aiming to high-accuracy estimation, the proposed algorithm is based on instantaneous voltage estimation and current measurements. Therefore, its dynamic is very close to that of the current control loop. After commissioning, the torque control loop, closed on the estimated torque feedback, features a bandwidth of about $800 \mathrm{~Hz}$, practically the same as that of the current 
Table III

BASE VALUES FOR NORMALISATION.

\begin{tabular}{c|c}
\hline Speed $\Omega_{B}$ & $5200 \mathrm{rpm}$ \\
\hline Torque $T_{B}$ & $112 \mathrm{Nm}$ \\
\hline Nominal phase voltage $U_{B}$ & $286 \mathrm{~V}$ \\
\hline Pole pairs $p$ & 3 \\
\hline Current $I_{B}$ & $2 T_{B} \Omega_{B} /\left(3 U_{B}\right)$ \\
\hline Flux linkage $\Lambda_{B}$ & $p U_{B} / \Omega_{B}$ \\
\hline Inductance $L_{B}$ & $\Lambda_{B} / I_{B}$ \\
\hline Resistance $R_{B}$ & $U_{B} / I_{B}$ \\
\hline Viscous coefficient $B_{B}$ & $p T_{B} / \Omega_{B}$ \\
\hline Inertia $J_{B}$ & $p B_{B} / \Omega_{B}$ \\
\hline Angle $\vartheta_{B}$ & $1 \mathrm{rad}$ \\
\hline Time $t_{B}$ & $1 / \Omega_{B}$ \\
\hline
\end{tabular}

loop. Figure 14 reports a sinusoidal torque reference and the relative estimated torque. The reference amplitude is 0.5 p.u. with a frequency of $1 \mathrm{kHz}$, obtained at a motor speed of 0.6 p.u.. The phase shift is slightly less than $1 \mathrm{rad}$.

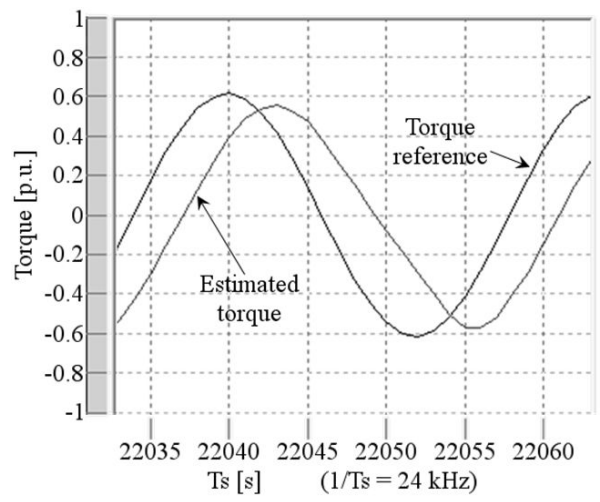

Figure 14. Reference torque vs. estimated torque.

\section{CONCLUSIONS}

The paper reports an instantaneous shaft torque estimator and the related commissioning procedure for its tuning, for PMSM drives. The commissioning is performed off-line and it is based on input power measurements, with a comprehensive view of all different torque contributions. The experiments performed in laboratory have confirmed a relative accuracy better than $4 \%$, and excellent dynamic performances.

\section{APPENDIX}

Normalised (p.u. values) are obtained by dividing each variable of interest by the corresponding base value. Primary base quantities are the torque $T_{B}$, the speed $\Omega_{B}$ and the nominal phase voltage $U_{B}$. They can be used to obtain the derived base quantities as reported in Table III.

\section{REFERENCES}

[1] D. M. Ionel, M. Popescu, M. I. McGilp, T. J. E. Miller, and S. J. Dellinger, "Assessment of torque components in brushless permanentmagnet machines through numerical analysis of the electromagnetic field," IEEE Trans. Ind. Appl., vol. 41, no. 5, pp. 1149-1158, Sep./Oct. 2005 .
[2] Y. Ohba, S. Masaki, K. Ohishi, T. Asai, K. Majima, Y. Yoshizawa, and K. Kageyama, "Sensorless force control for injection molding machine using reaction torque observer considering torsion phenomenon," IEEE Trans. Ind. Electron., vol. 56, 2009, accepted for future publication.

[3] Y. A.-R. Mohamed, "A hybrid-type variable-structure instantaneous torque control with a robust adaptive torque observer for a highperformance direct-drive PMSM," IEEE Trans. Ind. Electron., vol. 54, no. 5, pp. 2491-2499, Oct. 2007.

[4] T.-J. Ren and T.-C. Chen, "Modelling and control of a power-assisted mobile vehicle based on torque observer," IET Control Theory \& Applications, vol. 1, no. 5, pp. 1405-1412, Sep. 2007.

[5] S. Katsura and K. Ohnishi, "Human cooperative wheelchair for haptic interaction based on dual compliance control," IEEE Trans. Ind. Electron., vol. 51, no. 1, pp. 221-228, Feb. 2004.

[6] Y. Liu, Z. Q. Zhu, and D. Howe, "Instantaneous torque estimation in sensorless direct-torque-controlled brushless DC motors," IEEE Trans. Ind. Appl., vol. 42, no. 5, pp. 1275-1283, Sep./Oct. 2006.

[7] S.-K. Chung, C.-S. Kim, C.-G. Kim, and M.-J. Youn, "A new instantaneous torque control of PM synchronous motor for high-performance direct-drive applications," IEEE Trans. Power Electron., vol. 13, no. 3, pp. 388-400, May 1998.

[8] J.-X. Xu, S. K. Panda, Y.-J. Pan, T. H. Lee, and B. H. Lam, "A modular control scheme for PMSM speed control with pulsating torque minimization," IEEE Trans. Ind. Electron., vol. 51, no. 3, pp. 526-536, Jun. 2004.

[9] D. S. Reay, "CMAC and B-spline neural networks applied to switched reluctance motor torque estimation and control," in Proceedings of the 29th annual conference of the IEEE Industrial Electronics Society (IECON 2003), vol. 1, Roanoke, VA, USA, Nov. 2003, pp. 323-328.

[10] F. V. de Carvalho, L. E. B. da Silva, J. O. P. Pinto, B. K. Bose, and G. L. Torres, "A DSP based torque meter for induction motors," in Proceedings of the 29th annual conference of the IEEE Industrial Electronics Society (IECON 2003), vol. 1, Roanoke, VA, USA, Nov. 2003, pp. 414-418.

[11] B. K. Bose and N. R. Patel, "Programmable cascaded low-pass filterbased flux synthesis for stator flux-oriented vector-controlled induction motor drive," IEEE Trans. Ind. Electron., vol. 44, no. 1, pp. 140-143, Feb. 1997.

[12] G. Rizzoni, Y. Guezennec, A. Soliman, and B. Lee, "Engine control using torque estimation," US Patent 6866024, Mar. 15, 2005.

[13] P. Gyan, S. Ginoux, J. C. Champoussin, and Y. Guezennec, "Crankangle based torque estimation: mechanistic/stochastic," SAE technical paper 2000-01-0559, 2000.

[14] N. Urasaki, T. Senjyu, and K. Uezato, "A novel calculation method for iron loss resistance suitable in modeling permanent-magnet synchronous motors," IEEE Trans. Energy Convers., vol. 18, no. 1, pp. 41-47, Mar. 2003.

[15] _ "An accurate modeling for permanent magnet synchronous motor drives," in Proceedings of the 15th annual IEEE applied power electronics conference and exposition (APEC 2000), vol. 1, New Orleans, LA, USA, Feb. 2000, pp. 387-392.

[16] S. Morimoto, Y. Tong, Y. Takeda, and T. Hirasa, "Loss minimization control of permanent magnet synchronous motor drives," IEEE Trans. Ind. Electron., vol. 41, no. 5, pp. 511-517, Sep./Oct. 1994.

[17] N. Urasaki, T. Senjyu, and K. Uezato, "Relationship of parallel model and series model for permanent magnet synchronous motors taking iron loss into account," IEEE Trans. Energy Convers., vol. 19, no. 2, pp. 265-270, Jun. 2004.

[18] A. Ruderman and R. W. Jr., "Electrical machine PWM loss evaluation basics," in Proceedings of 4th international conference on energy efficiency in motor driven systems (EEMODS 2005), vol. 1, Heidelberg, Germany, Sep. 2005, pp. 58-68.

[19] J. Hu and B. Wu, "New integration algorithms for estimating motor flux over a wide speed range," IEEE Trans. Power Electron., vol. 13, no. 5, pp. 969-977, Sep. 1998. 


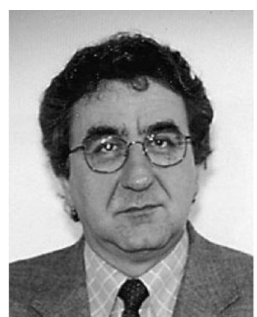

Silverio Bolognani (M'76) received the Laurea degree in electrical engineering from the University of Padova, Padova, Italy, in 1976. In the same year, he joined the Department of Electrical Engineering, University of Padova, where he is currently a Full Professor of electrical converters, machines, and drives. He started with the Electrical Drives Laboratory, University of Padova, where his research on brushless and induction motor drives was carried out in the framework of European and national research projects. He is currently engaged in research on advanced control techniques for motor drives and motion control and on design of AC electrical motors for variable-speed applications. He is the author of three patents and more than 200 papers on electrical machines and drives. Prof. Bolognani is currently the Chairman of the IEEE North Italy IAS/IES/ PELS Joint Chapter. He has been serving international conferences as a member of the Steering or Technical Committees as well as an Invited Speaker.

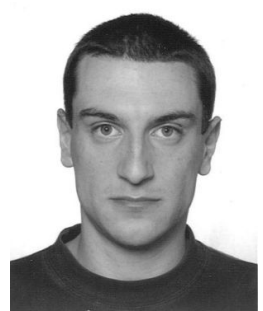

Luca Peretti (S'07) received the M.Sc. degree in electronic engineering from the University of Udine, Italy, in 2005, and the Ph.D. in mechatronics and industrial systems from the University of Padova, Italy, in 2009. From November 2007 to March 2008 he has been a visiting Ph.D. student at ABB Corporate Research Center, Department of Power Technologies, Västerås, Sweden. From January 2009 he is helding a post-doctoral research position at the Department of Technique and Management of Industrial Systems, University of Padova, Vicenza, Italy. His main research activity concerns advanced sensorless control and parameter estimation techniques for electrical motor drives.

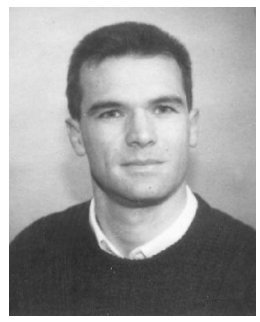

Mauro Zigliotto (M'88) received the Laurea degree in electronic engineering from the University of Padova, Padova, Italy, in 1988. He worked in industry as an R\&D Manager, developing DSP-based control systems for electric drives. From 1992 to 1999 , he was a Senior Research Assistant with the Electric Drives Laboratory, University of Padova. In 2000, he joined the Department of Electrical, Management and Mechanical Engineering, University of Udine, Udine, Italy, as an Associate Professor of electric drives. Since November 2005, he has been with the Department of Technique and Management of Industrial Systems, University of Padova, Vicenza, Italy, where he started working in the Electric Drives Laboratory. His main research interests include advanced control strategies for ac motors, and he has published extensively in this area. Prof. Zigliotto is currently the Secretary of the IEEE IAS/IES/PELS North Italy Joint Chapter. $\mathrm{He}$ has been serving international conferences as a member of the Steering or Technical Committees.

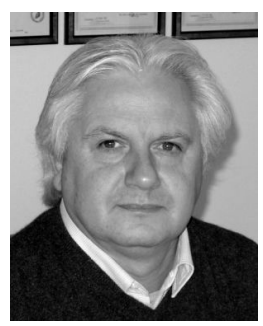

Ezio Bertotto is native of Venezia, Italy. From 1973 to 1978 he worked for BMB in the field of industrial electronics. In 1978, he participated in the foundation of the company EEI srl as a minority member. In 1982, he has been the reference member for the foundation of the company SCE srl. In 1984, he participated in the foundation of the company REEL srl (electrical drive manufacturer) as the majority chartered member, and in the foundation of the company ITACO srl (industrial automation operator) with other chartered members. Mr. Bertotto is currently leading REEL srl and ITACO srl. 